\title{
Metamatrial Based Packaging Method for Improved Isolation of Circuit Elements in Microwave modules
}

\author{
Ashraf Uz Zaman, Mubarak Sani Ellis, Per-Simon Kildal \\ Department of Signals and Systems \\ Chalmers University of Technology \\ SE-41296, Göteborg, Sweden \\ zaman@chalmers.se ; per-simon.kildal@chalmers.se
}

\begin{abstract}
In this work, periodic structure has been used to design a new type of packaging technique to improve the isolation between microwave components within a module. In contrast to the conventional packaging technique, the new technique relaxes the requirement for using vias in the substrate and using conductive adhesive or gasket material within a typical multiple compartment microwave modules. On the other hand it uses a periodic structure of metal pins to form a lid for the microwave module. The achieved level of isolation in $\mathrm{X}$-band and Ku-band for a two compartment test circuit is better than $65 \mathrm{~dB}$ which is better than the existing packaging technique within the band of interest.
\end{abstract}

Keywords- packaging method, mult-compartment microwave module, iolation, electromagnetic interference, periodic structure.

\section{INTRODUCTION}

Reliable and cost effective radio frequency (RF) packaging is becoming more and more important due to the significant growth of high frequency commercial applications in microwave communication and sensing. To comply with the smaller size requirements and compactness for mm-wave microwave modules, large amount of electronic components are to be placed into a confined area. Thus one of the most challenging design aspects of high frequency RF packaging is to maintain high isolation among the adjacent microwave circuit elements within a microwave module [1-3]. In conventional packaging technology as shown in figure 1, a rectangular cavity formed by metal enclosure is usually placed on top of a circuit element so as to suppress any possible interference with the nearby circuit components. Usually, this packaging system also consists of a conductive gasket or conductive adhesive material. The primary function of these gaskets or conductive adhesive is to provide sufficient electrical conductivity across the metal enclosure junction. Also, there is a strong requirement of putting via holes on the substrate around the circuit element to suppress the spurious modes propagating through the substrate [3-4]. Other possibilities of suppressing the substrate leaky modes are more complicated techniques such as insertion of doped silicon $(\mathrm{Si})$ sub-mount layer (high resistivity layer), tailoring of the substrate thickness to stop the power coupling to the $T M_{0}$ mode. But absolute shielding is almost impossible in most of these cases. This is because, the signal lines that connect the circuits inside the package are usually run through side wall mouse hole type opening shown in figure 1. Depending upon the size of the holes, they can cause substantial degradation in isolation [2].

Therefore, an ideal packaging solution will be one which avoids lot of vias in the substrate and avoids conductive adhesive or gasket along with the metal enclosure. The degradation of isolation due to the small opening for running signal lines from one compartment to another should also be avoided. All possible cavity modes should also be suppressed by the packaging enclosure within the band of interest [5].

With these things in mind, a new packaging or shielding method based on newly proposed gap waveguide technology has been used to have a good isolation among adjacent microwave circuit components. This gap waveguide technology makes use of parallel plate stop-band obtained by a smooth metal plate and a metal plate with a grid of pins as shown in figure 2 and is well presented in several research articles [6-8].

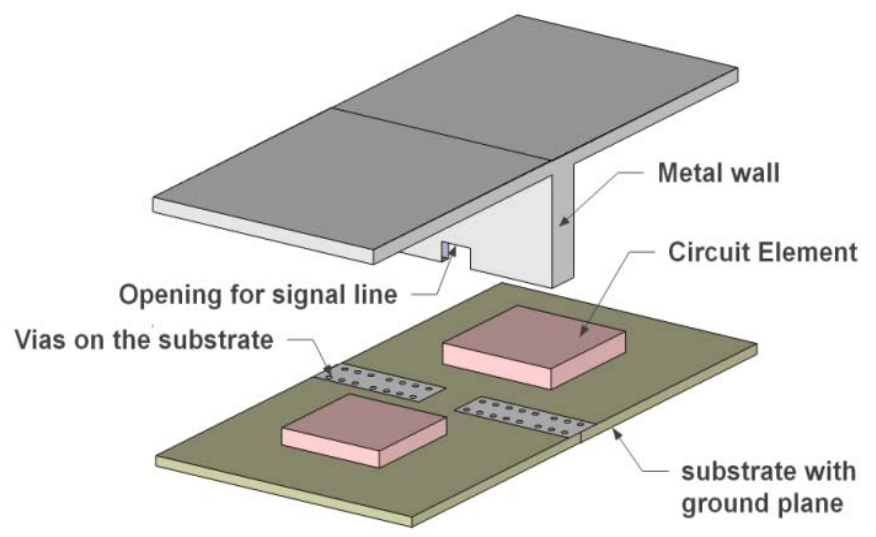

Figure 1. Conventional metal wall shielding with vias on substrate

This gap waveguide technology has the potential to be used as a low loss transmission line and good performance passive microwave components can be designed easily with this technology as mentioned in [9-10]. Also controlling of unwanted parallel plate modes within a specific bandwidth makes it very suitable for RF packaging application. The packaging aspect of gap waveguide technology has been demonstrated earlier for purpose of suppressing cavity modes and unwanted radiation in passive microstrip circuits [11-13]. 

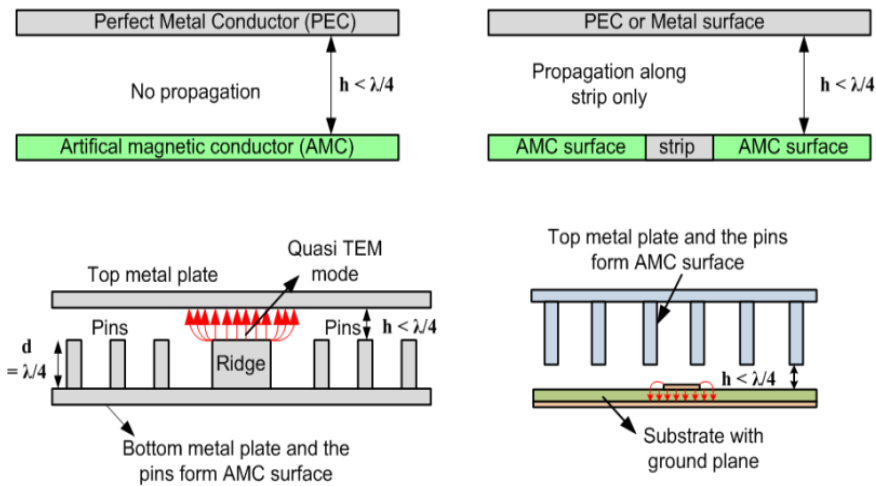

Figure 2. Gap waveguide technology

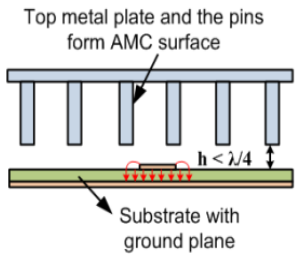

Usually, the parallel plate stop-band obtained for this gap waveguide technology is dependent on how the AMC surface is realized in practical applications [11], [14]. If the AMC is realized by a grid of metal pins, the air gap ' $h$ ' between the pin lid and the microstrip substrate plays an important role in determining the stop-band over which no unwanted mode can propagate. Smaller the air gap, the larger is the stop-band and more suitable it becomes for packaging application.

Now it is clear from the above discussion that - the isolation in case of gap waveguide packaging technology is not dependent on very good metal contact between the circuit board and the metal enclosure, so to say no proper grounding is needed. Thus, this technology can relax the requirement of using conductive adhesive or gasket material for multicompartment microwave modules. Also, the surface waves propagated through the substrate will be stopped without putting any grounded vias. This can save space and allow accommodation of more circuit elements within a microwave module in a more compact form.

\section{TEST CIRCUIT AND DESIGN OF THE PIN LID PACKAGE}

Two simple microstrip circuits on a typical Rogers 3003 substrate with thickness $0.254 \mathrm{~mm}$ was used as test circuits. Both the test circuits had two rectangular microstrip patches and one of them having grounded vias through the substrate. These two test circuits were analyzed with conventional two compartment metal enclosure type package and proposed gap waveguide package. The two test cases are shown in fig 3 (a) and $3(b)$.

The separation between the microstrip patches was kept to be $10 \mathrm{~mm}$ and these patches were fed by two $50 \Omega$ lines. For the conventional metal packaging, the height of the package was fixed at $2 \mathrm{~mm}$. It is important to note that no opening of sidewall was allowed in this test circuit for signal lines. So, the conventional metal wall package was considered to be completely shielded. On the other hand, the gap waveguide packaging had an allowable air gap between the metal pin and the substrate. So, there was no problem of running signal lines from one component to other one. This would allow signal propagation from one circuit element to another without deteriorating the isolation between the components. Also, in case of gap waveguide packaging technique, the substrates did not have any grounded vias to stop the surface modes. In this particular work, the completely shielded metal packaging was taken as a reference case and a comparison between the existing packaging technique and gap waveguide technique is presented.

For the gap waveguide pin lid case, two designs had been made at X-band and $\mathrm{Ku}$ - band. Initially, the cut-off properties of a lid of nails over a grounded substrate without microstrip lines were studied. Then the design rules mentioned in [14] were followed for designing the pin size and period of the pins at these two frequency band. For X-band, chosen values of ' $a$ ', ' $d$ ' and ' $p$ ' were $5.6 \mathrm{~mm}, 9 \mathrm{~mm}$ and $13 \mathrm{~mm}$ respectively. For Ku-band, these were $3 \mathrm{~mm}, 5 \mathrm{~mm}$ and $7.5 \mathrm{~mm}$ respectively. The height of the allowable air gap ' $h$ ' was chosen to be 3.0 $\mathrm{mm}$ (for X-band) and $2.0 \mathrm{~mm}$ (for Ku-band) respectively. The air gap height ' $h$ ' was chosen in a way to allow typical surface mount components (height around $1.7 \mathrm{~mm}$ ) to be placed on the circuit board. The choosing these particular heights values would reduce the overall stop band of the pin structure but still provided a considerable bandwidth.

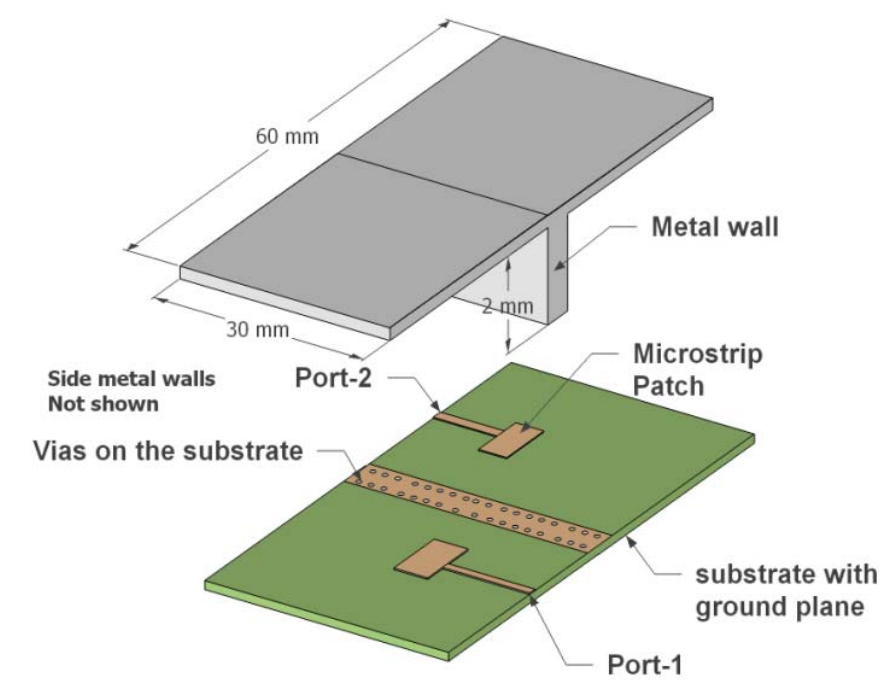

Figure 3(a). Test circuit for conventional metal packaging

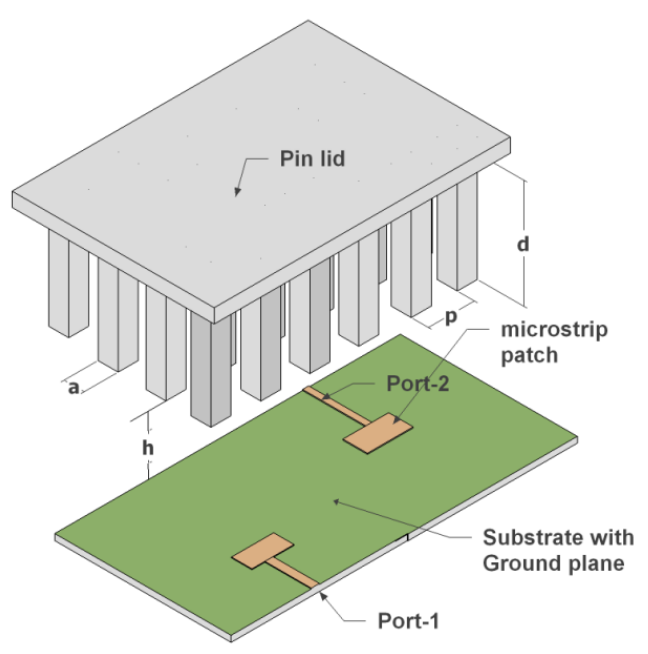

Figure 3(b). Test circuit for pin lid packaging technique 


\section{SIMULATION AND MEASUREMENT RESULTS}

To test the leakage from one side of the package to the other, two microstrip patches were used to measure the coupling between the two patches. Using the network analyzer, two port measurements were made and $S_{21}$ was compared for the conventional case and proposed pin lid packaging case in $\mathrm{X}$ band and $\mathrm{Ku}-\mathrm{Band}$. Also, for the proposed pin lid package, measurements were done in $\mathrm{Ku}$ band to check the performance with and without the presence of grounded via. The fig. 4 and fig. 5 shows the simulated and measured results respectively. Fig. 6 shows the performance without grounded vias for the pin lid case in comparison to metal wall without vias. The manufactured test circuits and the packaging enclosures are shown in fig.7.

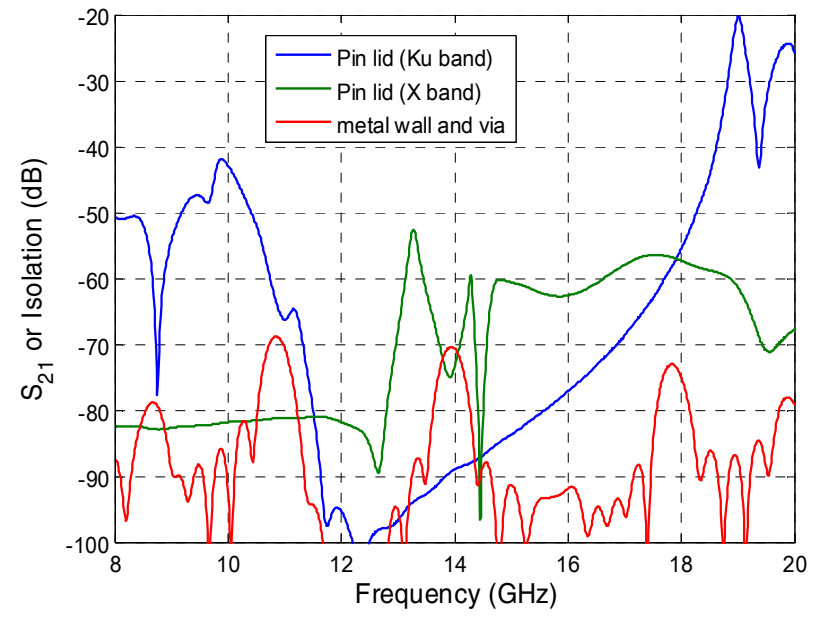

Figure 4. Simulated results for conventional packaging and proposed pin lid packaging.

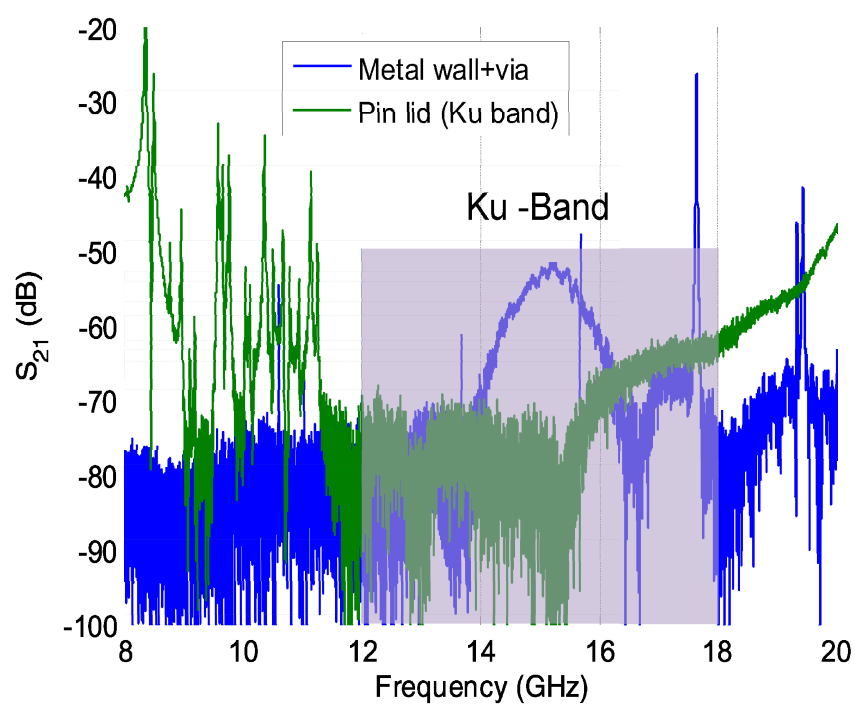

Figure 5 (a). Measured isolation results for metal wall package and $\mathrm{Ku}$ band pin lid package

As seen from the measurement results of figure 5, the two pin lid design presented relatively better isolation than that of a conventional metal wall packaging over the specified frequency bands. Isolation better than $65 \mathrm{~dB}$ and $60 \mathrm{~dB}$ was achieved for $\mathrm{X}$-band design and $\mathrm{Ku}$-band design respectively. Also, fig.6 clearly shows the need of vias in case of conventional metal wall package, whereas for the proposed pin lid case the grounded vias did not play any role in performance. Another significance of the proposed gap waveguide packaging technique is that- it does not depend on dimension of the circuit board. Once designed for a particular band of interest, good isolation is achieved over that band irrespective of cavity size or circuit board size. But it is to be noted that- the isolation performance for the proposed gap waveguide or pin lid packaging technique is very much related to the parallel-plate stop band obtained by the texture of metal pin surface. Outside this stop-band, the parallel plate modes can propagate easily and isolation between the adjacent circuits is destroyed. Typically, for a metal pin surface, this stop-band is achieved over a relative bandwidth of $60 \%$ [6], [11]. But in this case, due to the presence of dielectric substrate and a fixed allowable air gap height ' $h$ ', the relative bandwidth is reduced to $40 \%$ which still is quite significant for many microwave applications.

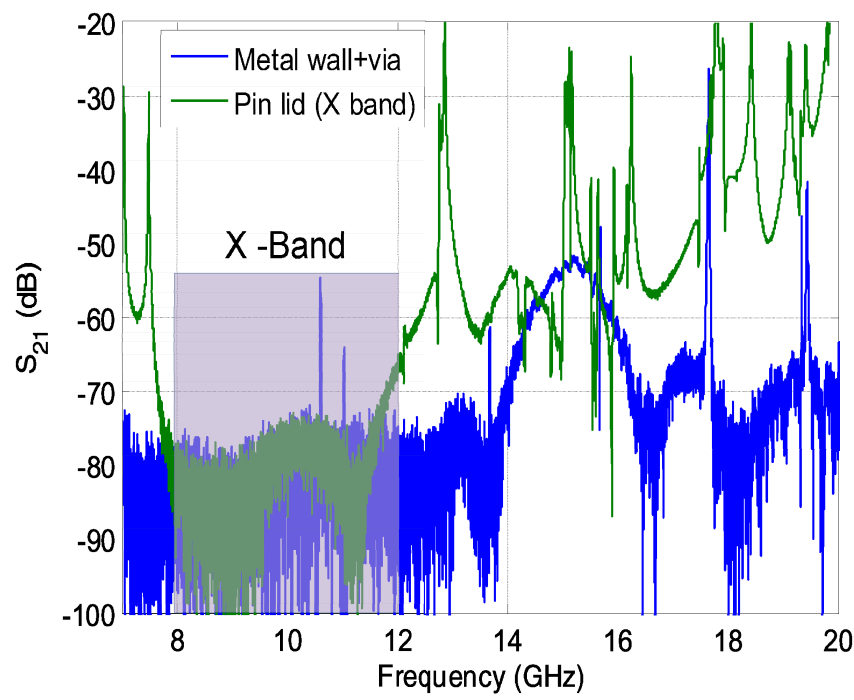

Figure 5 (b). Measured isolation results for metal wall package and Xband pin lid package

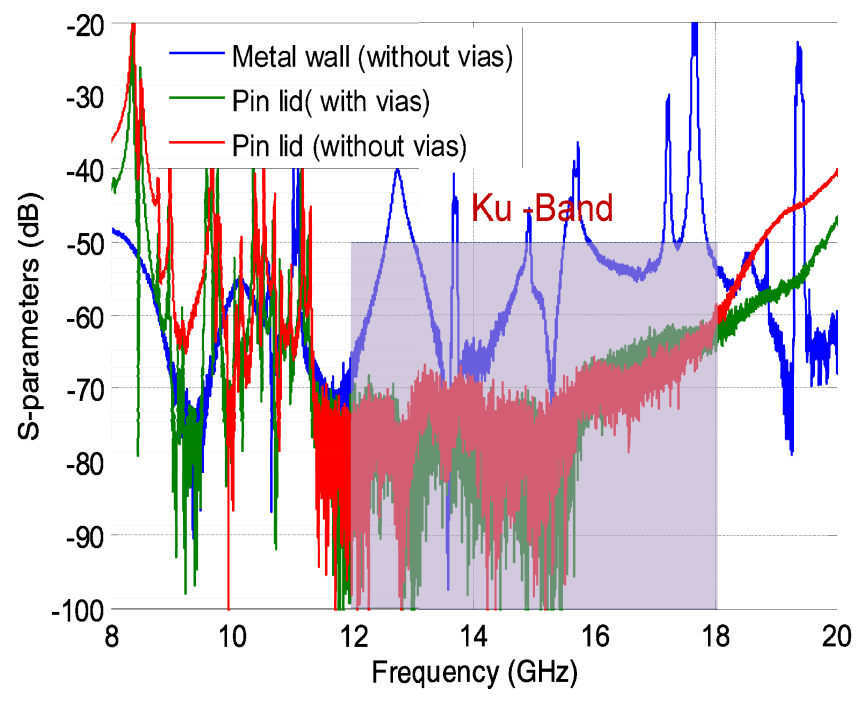

Figure 6. Effect of grounded vias 
It is to remember that, conventional metal wall packaging tested in this work is an ideal one without any side wall hole for signal lines. With the option of side wall hole present, the isolation is expected to deteriorate more. In that case, the proposed gap waveguide packaging technique will offer better isolation with the option of possible signal lines between the circuit elements.

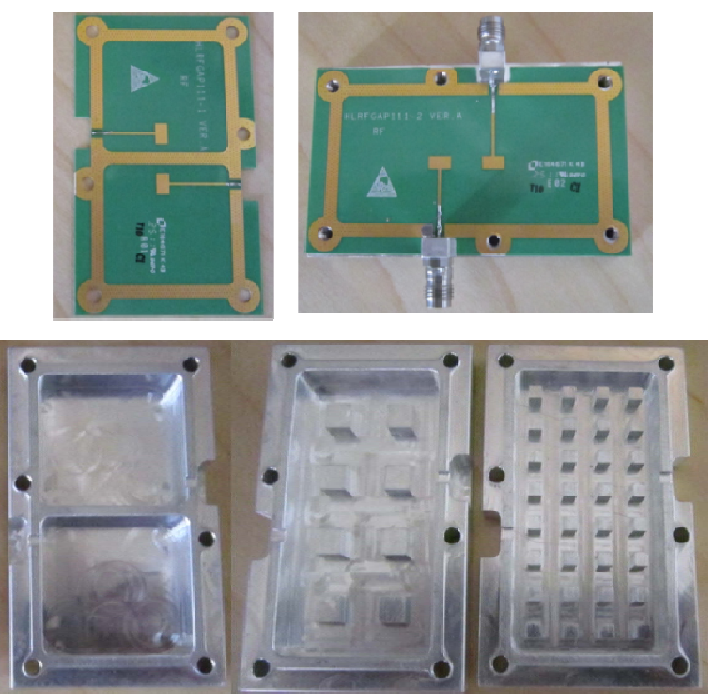

Figure7. Manufactured PCBs with patches and different packaging enclosure

\section{CONCLUSION}

In this paper, a new packaging technique based on gap waveguide technology has been proposed for improved isolation performance among various circuit elements within a microwave module. The new packaging technology uses a metal enclosure with uniform grid of pins instead of a smooth metal wall of conventional packaging method. This uniformly placed metal pins along with the ground plane of the substrate create a parallel-plate stop-band over which the isolation between the adjacent circuit elements are maximum. In this work, the isolation was measured between two side by side microstrip patches while separating the patches with conventional metal wall plus grounded via based packaging solution and also with proposed gap waveguide packaging solution. The measured results obtained for two different frequency bands such as X-band and Ku-band prototype agree quite well with the simulated performances. The proposed pin lid based gap waveguide packaging solution offers isolation level better than the conventional packaging solution. It has been proven to have the potential to stop unwanted leakage via the substrate as well as leakage by unwanted coupling over the air. It also relaxes the need for using conductive adhesive for making good grounding and the visas to suppress the surface waves propagating through the substrate. More significantly, the dimension of the cavity or circuit board size does not play any role in isolation performance for the proposed gap waveguide packaging solution. As a result, the proposed gap waveguide packaging enclosure can be an attractive packaging solution for low cost high frequency multi-compartment microwave module where the pin sizes will not be too big to handle.

\section{ACKNOWLEDGEMENT}

The authors would like to thank Ou Jian of Huawei Technologies, Sweden for his valuable discussion during this work and manufacturing of the prototypes.

\section{REFERENCES}

[1] T.A Midford, John J. Wooldridge, Rich L. Sturdivant "Evolutions of Packages for Monolithic Microwave and Millimeter Wave Circuits," IEEE Transection o Antennas and Propagation, Vol.43, No.9, pp. 983991, September 1995.

[2] L.Schulwitz, S.Ortiz, A. Mortazawi, "Packaging Method for Increased Isolation Using a Mcirostrip to Waveguide Transition," IEEE Microwave and Wireless Component Letters, Vol.17, No.3, pp.163-165, March 2007.

[3] A.Margomenos, K.J.Herrick, M.I.Herman, S.Valas, and L. P.B.Katehi, "Isolation in Three-Dimensional Integrated Circuits," IEEE Trans. Micro. Theory and Techniques, vol. 51, no.1, pp. 25-32, January 2003.

[4] G.E. Ponchak, D. Chun, J.-G. Yook and L.P.B.Katehi, "The Use of Metal filled Via Holes for Improving Isolation in LTCC RF and Wireless Multichip Packages," IEEE Trans. Advanced Packaging, vol. 23, no.1, pp. 88-99, February 2000.

[5] D. f. Williams, "Damping of the Resonant Modes of a Rectangular Metal Package", IEEE Transection on Microwave Theory and Techniques, Vol. 37, No. 1, pp.253-256, January 1989

[6] P.-S. Kildal, A. Uz Zaman, E. Rajo-Iglesias, E. Alfonso, A. ValeroNogueira, "Design and experimental verification of ridge gap waveguides in bed of nails for parallel plate mode suppression", IET Microwave, Antennas \& Propagation, Vol. 5, No. 3, pp. 262-270, March 2011.

[7] P.-S. Kildal, E-Alfonso, A. Valero-Nogueira, E. Rajo-Iglesias, "Local metamaterial-based waveguides in gaps between parallel metal plates" IEEE Antennas and Wireless Propagation Letters, vol. 8, pp. 84-87, 2009.

[8] A. Uz. Zaman, P.-S. Kildal, M. Ferndahl, A. Kishk, "Validation of Ridge Gap Waveguide Performance Using in-house TRL Calibration Kit", 4th European Conference on Antennas and Propagation, EuCAP 2010, Barcelona, April 2010.

[9] A. Uz. Zaman, P.-S. Kildal and A. Kishk, “ Narrow-Band Microwave Filter Using High-Q Groove Gap Waveguide Resonators with Manufacturing Flexibility and no Sidewalls", Accepted for publication in IEEE Transection of Components, Packaging and Manufacturing Technology.

[10] A. Uz. Zaman and P.-S. Kildal, "Slot Antenna in Ridge Gapwaveguide Technology", 6th European Conference on Antennas and Propagation EUCAP 2011, Prague, 25-29 March, 2012.

[11] E. Rajo-Iglesias, A. Uz Zaman, P.-S. Kildal, "Parallel plate cavity mode suppression in microstrip circuit packages using a lid of nails", IEEE Microwave and Wireless Components Letters, Vol. 20, No. 1, pp. 31-33, Dec. 2009.

[12] A.Uz. Zaman, J.Yang, P.-S. Kildal, "Using Lid of Pins for Packaging of Microstrip Board for Descrambling the Ports of Eleven Antenna for Radio Telescope Applications", IEEE Antennas and Propagation Society International Symposium, July 2010.

[13] A. Algaba Brazález, A. Uz Zaman, E. Pucci, E. Rajo-Iglesias, P.-S. Kildal, A. Kishk,'Improving microstrip filters with gap waveguide packaging", 5th European Conference on Antennas and Propagation EUCAP 2011, Rome, Italy, 11-15 April 2011.

[14] E. Rajo-Iglesias and P.-S. Kildal, "Numerical studies of bandwidth of parallel plate cut-off realized by bed of nails, corrugations and mushroom-type EBG for use in gap wave-guides", IET Microwaves, Antennas \& Propagation, Vol.5, Iss.3, pp. 282-289, March 2011. 